\title{
Phase Stability, Phase Transformations, and Reactive Phase Formation
}

\section{Sinn-wen Chen}

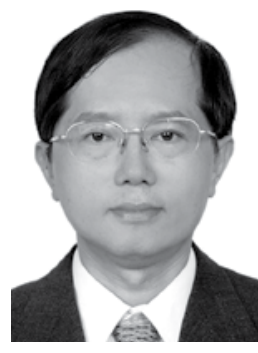

This special topic in JOM, Phase Stability, Phase Transformations, and Reactive Phase Formation, is sponsored by the Alloy Phases Committee and

presents five articles.

In the first paper, K. Suganuma and coworkers, from Osaka University, Japan, give a comprehensive overview of high-temperature lead-free solders including their properties and phase formation. High-temperature, lead-free soldering is a key technology for electronics components and assemblies, and requires a high level of process control. The interconnections must withstand temperatures beyond $250^{\circ} \mathrm{C}$ for a short period and offer high reliability against thermal cycling as well as good heat dissipation. Therefore, a robust design concept is needed based on materials science in order to obtain the new technology.

Y.-C. Wu et al., from National Tsing Hua University, Taiwan, study phase ordering transformation of $\mathrm{L}_{0}$ ordered FePt film by controlling stress and diffusion. The ordering process of a disordered FePt is controlled by a kinetic process. The paper proposes several ways to accelerate phase transformation at low temperature. An extra driving force for diffusion was provided by ion irradiation or dynamic stress; the diffusion length was diminished by using atomic-scale multilayer deposition. In addition, the novel sputtering scheme, atomic-scale multilayer deposition, can effectively promote the (001) texture of $\mathrm{L}_{0}$ FePt grown on amorphous substrates without any underlayer
J.P. Chu from National Taiwan University of Science and Technology, Taiwan, reports an interesting phase transformation phenomenon, annealing-induced amorphization in a copper-based $\left(\mathrm{Cu}_{51} \mathrm{Zr}_{42} \mathrm{Al}_{4} \mathrm{Ti}_{3}\right)$ glass-forming thin film. Chu found that upon annealing in the supercooled liquid region, a fully amorphous structure is developed and this leads to a softening

\section{These five articles \\ clearly indicate that \\ "phases" are \\ important for all kinds \\ of materials including \\ structural materials, \\ magnetic materials, glasses, and electronic \\ materials.}

in hardness and an abrupt increase $(\sim 25$ times) in a decreasing trend of electrical resistivity.

V.I. Dybkov, from the Institute of Problems of Materials Science, Ukraine, studies the fundamentals of the reactive phase formation in solder joints. In the course of soldering, intermetallic layers usually occur at the solid metal-liquid solder interface, while the solid simultaneously dissolves in the solder melt. The interconnection of these two processes is considered in this paper.

I. Calliari et al., from the University of Pad ova, Italy, investigates the phase transformation during continuous cooling and isothermal treatments of commercial duplex stainless steels. Stainless steels, especially the grade used in this study, suffer from lower toughness caused by secondary phase precipitation especially when these steels undergo thermal treatments. In this study the influence of thermal treatment parameters on the precipitation of these secondary phases was investigated.

A phase is a homogeneous region of matter and is the building block of materials. Different spatial arrangements of molecules lead to different phases and result in different materials properties. These five articles clearly indicate that "phases" are important for all kinds of materials including structural materials, magnetic materials, glasses, and electronic materials. It can be noticed as well that the contributors are from different regions of the world. As one of the oldest committees in TMS, the Alloy Phases Committee clearly indicates the very early awareness of the importance of "phase" in material studies by the TMS members.

The Alloy Phases Committee has also sponsored a Phase Stability, Phase Transformations, and Reactive Phase Formation in Electronic Materials symposium at the TMS Annual Meeting for eight consecutive years. In the foreword of the January 2009 Journal of Electronic Materials special issue, it is mentioned that the internationalized "phase" (相，そう, фаза, fase, faza) is the key word. The same globalized spirit should be shared. Although it might not necessarily get hotter and more crowded, the world of materials science has undoubtedly become more flattened, and global cooperation will be the key for the future.

Sinn-wen Chen is with the Department of Chemical Engineering, National Tsing Hua University, Hsin-chu, Taiwan and is the advisor to JOM from the Alloy Phases Committee of the Electronic, Magnetic, \& Photonic Materials Division of TMS. 